\title{
A nomogram to predict the risk of prolonged length of stay following primary total hip arthroplasty with an enhanced recovery after surgery program
}

Haosheng Wang ${ }^{1 \dagger}$, Tingting Fan ${ }^{2 \dagger}$, Wenle $\mathrm{Li}^{3,4 \dagger}$, Bo Yang ${ }^{5}$, Qiang Lin ${ }^{5}$ and Mingyu Yang ${ }^{1 *}$

\begin{abstract}
Background: The aim of this study was to identify the risk factors associated with prolonged length of stay (LOS) in patients undergoing primary total hip arthroplasty (THA) managed with an enhanced recovery after surgery (ERAS) program and develop a prediction model for improving the perioperative management of THA.
\end{abstract}

Methods: In this single-center retrospective study, patients who underwent primary THA in accordance with ERAS from May 2018 to December 2019 were enrolled in this study. The primary outcome was prolonged LOS (> $48 \mathrm{~h}$ ) beyond the first postoperative day. We collected the clinical patient's clinical characteristics, surgery-related parameters, and laboratory tests. A logistic regression analysis explored the independent risk factors for prolonged LOS. According to published literature and clinical experience, a series of variables were selected to develop a nomogram prediction model to predict the risk of prolonged LOS following primary THA with an ERAS program. Evaluation indicators of the prediction model, including the concordance index (C-index), the receiver operating characteristic (ROC) curve, calibration curve, and decision curve analysis, were reported to assess the performance of the prediction model. The bootstrap method was conducted to validate the performance of the designed nomogram.

Results: A total of 392 patients were included in the study, of whom 189 (48.21\%) had prolonged LOS. The logistics regression analysis demonstrated that age, sex, hip deformities, intraoperative blood loss, operation time, postoperative Day 1 (POD) hemoglobin (Hb), POD albumin (ALB), and POD interleukin-6 (IL-6) were independent risk factors for prolonged LOS. The C-index was 0.863 (95\% Cl 0.808 to 0.918 ) and 0.845 in the bootstrapping validation, respectively. According to the results of the calibration, ROC curve, and decision curve analyses, we found that the nomogram showed satisfactory performance for prolonged LOS in this study.

Conclusions: We explored the risk factors for prolonged LOS following primary THA with an ERAS program and developed a prediction model. The designed nomogram was expected to be a precise and personalized tool for predicting the risk and prognosis for prolonged LOS following primary THA with an ERAS program.

Keywords: Total hip arthroplasty, Length of stay, Enhanced recovery after surgery, Risk factors, Nomogram

*Correspondence: 616353108@qq.com

${ }^{\dagger}$ Haosheng Wang, Tingting Fan and Wenle Li have contributed to the work equally and should be regarded as co-first authors

1 Department of Orthopedics, Taizhou Central Hospital (Affiliated Hospital

To Taizhou College), Donghai Street, Taizhou 317700, Zhejiang Province, People's Republic of China

Full list of author information is available at the end of the article

\section{Background}

With the world's population aging, the demand for total hip arthroplasty (THA) is rising. Since the 1920s, THA has become one of the most effective treatments for many hip conditions. Enhanced recovery after surgery original author(s) and the source, provide a link to the Creative Commons licence, and indicate if changes were made. The images or other third party material in this article are included in the article's Creative Commons licence, unless indicated otherwise in a credit line to the material. If material is not included in the article's Creative Commons licence and your intended use is not permitted by statutory regulation or exceeds the permitted use, you will need to obtain permission directly from the copyright holder. To view a copy of this licence, visit http://creativecommons.org/licenses/by/4.0/. The Creative Commons Public Domain Dedication waiver (http://creativeco mmons.org/publicdomain/zero/1.0/) applies to the data made available in this article, unless otherwise stated in a credit line to the data. 
(ERAS) is a concept that has become increasingly popular for arthroplasty surgery over the last ten years [1-3]. The goals of ERAS are to promote faster recovery, reduce postoperative complications, shorten the length of stay (LOS), reduce the burden of medicine on society, and improve patient quality of life and satisfaction [4]. Henrik Kehlet [5], a Danish surgeon, has extensively addressed this complex pathophysiological phenomenon in the perioperative period since the late 1980s in collaboration with all surgical disciplines. As such, he used existing basic knowledge to change the perspectives in clinical practice. Today, the ERAS program has been considered an efficient and cost-effective use of health resources.

Notably, there is a huge difference between developed and developing countries in ERAS implementation. Therefore, based on the database of the project group for the "National Health and Family Planning Commission's public-benefit project: the safety and effect assessment of joint arthroplasty" and evidence-based medicine, a consensus (hereafter, "consensus") has been reached, to provide a reference for medical teams [6-9]. ERAS for patients undergoing primary THA has been in place in our hospital since May 2018. Painless surgery and fast recovery are common pursuits of surgeons and THA patients. Painless surgery and fast recovery are common pursuits of surgeons and THA patients. The focus of ERAS in TKA is to improve surgical techniques and optimize perioperative management, including the reduction of trauma and hemorrhage, optimization of pain and blood management, prevention of infection and deep vein thrombosis, and promotion of early mobilization. However, some patients are slow to discharge, which leads to delays [10]. This observation led us to investigate the related risk factors for prolonged LOS and reduce the costs, and in-hospital complications. At present, studies have not focused on establishing a prediction model for the risk of prolonged LOS after primary THA with an ERAS program. Therefore, it is necessary to fully understand the clinical characteristics of patients who underwent primary THA with an ERAS program to identify the risk factors for prolonged LOS. A well-developed clinical nomogram can be used to predict individual outcomes, which is beneficial to both patients and clinicians.

Thus, the aim of this study was to develop a predictive model by analyzing the data in our hospital to determine the risk for prolonged LOS following primary THA with an ERAS program.

\section{Methods}

\section{Inclusion and exclusion criteria}

Inclusion criteria: (1) Primary THA; (2) strict implementation of ERAS measures in consensus; (3) clear consciousness and no communication barriers; and (4) volunteered to participate. The exclusion criteria were as follows: (1) severe hematological diseases; (2) serious coexisting diseases such as heart, brain, and lung; and (3) mental illness or communication difficulties. This retrospective study was approved by the ethical and research committee of Taizhou Central Hospital (Project ID: $202009795 \mathrm{~N}$ ). All patients provided written informed consent.

\section{Patients and data collection}

A total of 392 patients who underwent primary THA strictly according to the ERAS consensus at the Department of Orthopedics, Taizhou Central Hospital from May 2018 to Dec 2019 were retrospectively reviewed. This retrospective study was approved by the ethical and research committee of Taizhou Central Hospital (Project ID: 202009795N). The primary THA operations were performed by the same surgeon following standard procedures through a posterolateral approach under general anesthesia. Preoperative pre-emptive analgesia usually chosen from NSAIDs or selective COX-2 inhibitors. Patients with high preoperative anxiety may be given diazepam and Stilnox and, if needed, the anxiolytic drugs olanzapine, escitalopram and sertraline. In addition, $15-20 \mathrm{mg} / \mathrm{kg}$ TXA was administered intravenously (IV) prior to skin incision and 1-2 g TXA was applied topically before closing the incision. No drainage tube was placed after the operation. Postoperative pain was treated with multimodal analgesics, including ice, NSAIDs, selective COX-2 inhibitors, morphine, pethidine and oxycodone. The specific use of analgesics can be adjusted by the attending physician according to the patient's specific condition.

Demographic and clinical parameters, including age, sex, body mass index (BMI), Harris score, proportion of hip deformities, hip joint mobility, and preoperative visual analogue score (VAS), were collected. Preoperative routine laboratory tests, included blood tests, blood electrolytes, liver function, and kidney function. Operation-related parameters including the American Society of Anesthesiologists (ASA) parameters, operation time, anesthesia time, intraoperative infusion volume, and intraoperative blood loss were recorded. A routine hematological examination was performed 1 day after surgery. Thus, we calculated the $\mathrm{Hb}$ change rate on Day 1 after surgery. $\mathrm{POD} \mathrm{Hb}$ change $=$ (preoperative $\mathrm{Hb}-\mathrm{POD} \mathrm{Hb}$ )/ preoperative $\mathrm{Hb} * 100 \%$, where POD represents one day after surgery. In parallel, we recorded the VAS score on the day of surgery and Day 1 after surgery, out-of-bed activity time after surgery, total infusion volume on the day of surgery, and postoperative infusion volume. In this study, patients with $\mathrm{LOS} \leq 48 \mathrm{~h}$ and $>48 \mathrm{~h}$ were the A and B groups, respectively. Moreover, parameters of 
postoperative complications, including postoperative nausea and vomiting (PONA), urinary tract infection, intermuscular vein thrombosis venous, deep vein thrombosis (DVT), delayed wound healing, superficial infection and deep infection, were collected.

\section{Discharge criteria and follow-up}

Patients who meet the following four criteria are judged to have recovered and can be released and discharged. (I): The patient's vital signs were stable, fever was not observed, spirit and appetite had returned to the preoperative level and stool was normal; (II) the incision was dry with no signs of infection, such as erythema and induration; and (III) hip pain after surgery was not obvious, could be effectively relieved by oral analgesics, did not affect the patient's sleep and functional exercises, and had a resting pain VAS score of $<3$ points and a pain VAS score of $<5$ points when active. The oral medications often used for postoperative pain are nonsteroidal antiinflammatory drugs (NSAIDs) or selective COX-2 inhibitors, such as diclofenac, loxoprofen sodium, celecoxib, and rofecoxib, combined with opioid analgesics for severe pain, including oral tramadol or oxycodone. After meeting the discharge criteria, patients were discharged home. Patient postoperative follow-up was conducted regularly at the outpatient department of Taizhou Central Hospital. Outpatient follow-up at was performed at 1 week, 2 weeks, 1 month, 2 months, 3 months, 6 months and 12 months after the operation. During the followup at 2 weeks after operation, the stitches were removed according to the healing of the incision, and venous thrombosis of the lower extremities was examined by color Doppler ultrasound.

\section{Statistical analysis and model construction}

Continuous variables are presented as the means \pm standard deviations or as medians and interquartile ranges (depending on the data distribution) and were evaluated using the Student's t test or Mann-Whitney U test, as appropriate. Categorical variables were grouped and compared using the $\chi^{2}$ test or Fisher's exact test. Forward step-wise multivariable logistic regression analysis, including covariates identified in univariate logistic analysis, was used to identify independent factors associated with prolonged LOS following primary THA with an ERAS program. Meanwhile, the odds ratio (OR) and 95\% confidence intervals (CIs) were reported. According to the results of the regression coefficients of independent variables, an individual nomogram prediction model for prolonged LOS was established. The designed nomogram model was internally validated using bootstrap sampling (1000 resamples). The area under the receiveroperating characteristic curve (AUC) or c statistic was used to assess the discrimination of the nomogram in receiver operating characteristic (ROC) curves. An AUC of 0.5 indicated no diagnostic performance; $0.5-0.7$ indicated inaccuracy in discrimination; $0.7-0.9$ indicated moderate performance; and $>0.9$ indicated excellent performance. Calibration was evaluated using a calibration plot to compare the relationship between the observed outcome frequencies versus the predicted outcomes. A decision curve analysis (DCA) was performed to assess the clinical net benefit. Analyses were performed with SPSS software (SPSS standard, version 26.0; SPSS, Inc.) and $\mathrm{R}$ version 4.0.1 (R Foundation for Statistical Computing). The nomogram was created in $\mathrm{R}$ software using the "rms" package. In this study, $P<0.05$ was considered statistically significant.

\section{Results}

Details of the patient population are shown in Table 1. A total of 392 patients were included, with 206 (52.6\%) males and 186 (47.4\%) females, the mean age $61.2 \pm 7.0$ years. Among the 392 patients, 203 patients had LOS $\leq 48$ h, i.e., Group A; 189 patients had LOS $>48$ h, i.e., Group B. Among the A and B group, there were statistically significant differences were observed in age $(P<0.001)$, sex $(P=0.04)$, hip deformities $(P=0.035)$, hip joint mobility $(P=0.008)$, preoperative VAS $(P=0.012)$, preoperative $\mathrm{Hb}(P=0.003)$, operation time $(P<0.001)$, anesthesia time $(P<0.001)$, intraoperative infusion volume $(P<0.001)$, out of bed activities time $(P<0.001)$, postoperative infusion volume $(P<0.001)$, total infusion volume on the day of surgery $(P<0.001)$, VAS score on the day of surgery $(P<0.001)$, POD VAS score $(P<0.001)$, POD ALB $(P<0.001)$, POD IL-6 $(P<0.001)$, POD total blood loss $(P=0.044)$, and POD Hb change rate $(P<0.001)$. Significant differences were not observed in remaining parameters between the A and B groups. Among the 392 patients in this study, we observed postoperative complications in both the A group (LOS $\leq 48 \mathrm{~h}$ ) and B group (LOS $>48 \mathrm{~h}$ ). Significant differences were not observed in PONA $(P=0.821)$, urinary tract infection $(P=0.031)$, intermuscular vein thrombosis venous $(P=0.245)$ or deep infection $(P=0.381)$ between the two groups. However, delayed wound healing, superficial infection, DVT, and total complications were significantly lower in the A group (LOS $\leq 48 \mathrm{~h}$ ) than B group (LOS $\leq 48 \mathrm{~h}$ ). Detailed data on the postoperative complications are presented in Table 2.

In the multivariate logistic regression, the following eight factors were independent risk factors for prolonged LOS (Table 3) $(P<0.05)$ : age (odds ratio $[\mathrm{OR}]=2.315$, 95\% confidence interval $[\mathrm{CI}]=1.490-3.140, P=0.042)$, sex (OR 0.305, 95\% CI 0.256-0.354, $P=0.039$ ), hip deformities (OR 0.296, 95\% CI 0.098-0.894, $P=0.031$ ), operation 
Table 1 Characteristic at baseline between A and B group

\begin{tabular}{|c|c|c|c|c|}
\hline & Total & A group & B group & $P$ value \\
\hline Number of patients & 392 & 203 & 189 & \\
\hline Age (years) & $61.2(7.0)$ & $58.0(6.8)$ & $64.6(5.5)$ & $<0.001$ \\
\hline \multicolumn{5}{|l|}{$\operatorname{Sex}(\%)$} \\
\hline Female & $186(47.4)$ & 75 (36.9) & $111(58.7)$ & \multirow[t]{2}{*}{0.04} \\
\hline Male & $206(52.6)$ & $128(63.1)$ & $78(41.3)$ & \\
\hline BMI $\left(\mathrm{kg} / \mathrm{m}^{2}\right)$ & $25.2(1.8)$ & $25.0(1.6)$ & $25.3(2.0)$ & 0.373 \\
\hline Harris score & $44.2(15.2)$ & $45.4(12.8)$ & $42.8(17.4)$ & 0.523 \\
\hline \multicolumn{5}{|l|}{ Hip deformities (\%) } \\
\hline No & $334(85.2)$ & $157(77.3)$ & $177(93.7)$ & \multirow[t]{2}{*}{0.035} \\
\hline Yes & $58(14.8)$ & $46(22.7)$ & $12(6.3)$ & \\
\hline Hip joint mobility $\left(^{\circ}\right)$ & $223.6(28.0)$ & $230.0(27.1)$ & $216.8(27.3)$ & 0.008 \\
\hline Preoperative VAS & $6.0(2.0)$ & $5.6(1.9)$ & $6.5(2.1)$ & 0.012 \\
\hline Preoperative Hb (g/L) & $134.7(16.8)$ & $139.0(12.9)$ & $130.0(19.2)$ & 0.003 \\
\hline Preoperative ALB (g/L) & $44.6(5.1)$ & $44.0(5.2)$ & $45.3(4.9)$ & 0.227 \\
\hline \multicolumn{5}{|l|}{ ASA (\%) } \\
\hline I & $18(4.6)$ & $15(7.4)$ & $3(1.6)$ & \multirow[t]{4}{*}{0.337} \\
\hline$\|$ & $213(54.3)$ & $119(58.6)$ & $94(49.7)$ & \\
\hline III & $150(38.3)$ & $66(32.5)$ & $84(44.4)$ & \\
\hline IV & $11(2.8)$ & $3(1.5)$ & $8(4.2)$ & \\
\hline Operation time (min) & $74.4(16.4)$ & $67.8(12.9)$ & $81.6(16.7)$ & $<0.001$ \\
\hline Anesthesia time (min) & $95.1(22.3)$ & $87.6(18.5)$ & $103.2(23.2)$ & $<0.001$ \\
\hline Intraoperative infusion volume (ml) & $372.0[334.0,438.0]$ & $337.0[317.0,354.0]$ & $440.0[414.0,475.0]$ & $<0.001$ \\
\hline Intraoperative blood loss (ml) & $182.0[146.0,210.0]$ & $182.0[151.0,203.0]$ & $183.0[145.0,222.0]$ & 0.442 \\
\hline Out of bed activities time (h) & $16.4[13.1,20.9]$ & $13.6[11.2,15.8]$ & $20.9[17.5,23.7]$ & $<0.001$ \\
\hline Postoperative infusion volume (ml) & $502.0[474.0,537.0]$ & $485.0[466.0,512.0]$ & $529.0[481.0,585.0]$ & $<0.001$ \\
\hline Total infusion volume on the day of surgery (ml) & $1050.1(127.4)$ & $962.4(80.1)$ & $1144.2(98.3)$ & $<0.001$ \\
\hline VAS score on the day of surgery & $2.8(0.9)$ & $2.5(0.9)$ & $3.2(0.8)$ & $<0.001$ \\
\hline Day 1 after surgery VAS score & $3.3(1.0)$ & $2.7(0.5)$ & $3.9(1.1)$ & $<0.001$ \\
\hline POD Hb (g/L) & $121.6(13.7)$ & $123.1(14.9)$ & $120.1(12.2)$ & 0.237 \\
\hline POD ALB (g/L) & $39.1(4.6)$ & $40.7(4.6)$ & $37.4(4.1)$ & $<0.001$ \\
\hline POD CRP (mg/L) & $18.2(5.2)$ & $18.3(5.4)$ & $18.1(5.1)$ & 0.936 \\
\hline POD IL-6 (pg/ml) & $20.8(8.1)$ & $16.5(6.4)$ & $25.5(7.2)$ & $<0.001$ \\
\hline POD total blood loss 1 day after surgery (ml) & $308.8(58.7)$ & $298.5(55.7)$ & $319.8(59.9)$ & 0.044 \\
\hline POD Hb change rate $(\%)$ & $9.0[8.0,11.0]$ & $8.0[6.0,9.0]$ & $10.0[9.0,12.0]$ & $<0.001$ \\
\hline
\end{tabular}

A group: LOS $\leq 48 \mathrm{~h}$ group; B group: LOS > $48 \mathrm{~h}$ group; BMI, body mass index; VAS: visual analogue score; Hb: hemoglobin; ALB: albumin; ASA: American Society of Anesthesiologists; CRP: C-reactive protein; IL-6: interleukin-6; POD: one day after surgery

$\mathrm{POD} \mathrm{Hb}$ change $=($ Preoperative $\mathrm{Hb}-\mathrm{POD} \mathrm{Hb}) /$ Preoperative $\mathrm{Hb} * 100 \%$

time (OR 1.135, 95\% CI 1.068-1.202, $P=0.008$ ), intraoperative blood loss (OR 1.798, 95\% CI 1.645-1.951, $P=0.028)$, POD $\mathrm{Hb}(\mathrm{OR} 0.955$, 95\% CI $0.924-0.987$, $P=0.006$ ), POD ALB (OR 0.689, 95\% CI 0.656-0.722, $P=0.026$ ), and POD IL-6 (OR 1.312, 95\% CI 1.063$1.620, P=0.011)$. Afterward, a nomogram was developed according to the logistic regression analysis (Fig. 1).

To evaluate the performance of the prediction model, several indicators were reported in this work. The area under the curve (AUC) of the receiver operating characteristic (ROC) curve (Fig. 2) of the model was 0.857 (95\%
CI, 0.815 to 0.898 ), indicating that the discrimination performance of the model was satisfactory. Additionally, the calibration curve demonstrated good agreement between the observed probability of prolonged LOS in this study (Fig. 3). Meanwhile, the C-index of the nomogram was 0.863 (95\% CI 0.808 to 0.918 ), while that by bootstrapping validation was 0.845 (bootstrap $=1000$ ). To identify the potential clinical benefit of the designed nomogram, a decision analysis (DCA) was performed in this dataset. The DCA is demonstrated in Fig. 4 and suggested that the clinical net benefit of this risk prediction 
Table 2 Comparison of variables between the A group and B group

\begin{tabular}{lllr}
\hline Parameters & A group $(\mathbf{n = 2 0 3 )}$ & B group $(\mathbf{n = 1 8 9 )}$ & $\boldsymbol{P}$ value \\
\hline Complications (\%) & & & \\
PONA & $11(5.42 \%)$ & $13(6.88 \%)$ & 0.821 \\
Urinary tract infection & $1(0.49 \%)$ & $4(2.12 \%)$ & 0.031 \\
Intermuscular vein thrombosis & $3(1.48 \%)$ & $3(1.59 \%)$ & 0.245 \\
DVT & $0(0.00 \%)$ & $4(2.12 \%)$ & 0.0165 \\
Delayed wound healing & $0(0.00 \%)$ & $5(2.65 \%)$ & 0.0071 \\
Superficial infection & $0(0.00 \%)$ & $6(3.17 \%)$ & $<0.001$ \\
Deep infection & $0(0.00 \%)$ & $1(0.53 \%)$ & 0.381 \\
Total & $15(7.39 \%)$ & $36(19.05 \%)$ & $<0.001$ \\
\hline
\end{tabular}

A group: LOS $\leq 48$ h group; B group: LOS > 48 h group; PONA: postoperative nausea and vomiting; DVT: deep vein thrombosis

Table 3 Univariate and multivariate logistic regression model analyses of prolonged LOS following primary THA with an ERAS program

\begin{tabular}{|c|c|c|c|c|}
\hline & \multicolumn{2}{|l|}{ Univariate analysis } & \multicolumn{2}{|c|}{ Multivariate analysis } \\
\hline & OR $(95 \% \mathrm{Cl})$ & $P$ value & OR $(95 \% \mathrm{Cl})$ & $P$ value \\
\hline Age (years) & $1.598(1.177-2.019)$ & 0.019 & $2.315(1.490-3.140)$ & 0.042 \\
\hline Sex $(\%)$ & $0.765(0.589-0.941)$ & 0.163 & $0.305(0.256-0.354)$ & 0.039 \\
\hline BMI (kg/m2) & $1.152(0.973-1.363)$ & 0.105 & NA & \\
\hline Harris score & $0.995(0.978-1.013)$ & 0.594 & NA & \\
\hline Hip deformities (\%) & $0.297(0.139-0.637)$ & 0.002 & $0.296(0.098-0.894)$ & 0.031 \\
\hline Hip joint mobility $\left(^{\circ}\right)$ & $1.625(1.013-2.237)$ & $<0.001$ & NA & \\
\hline Preoperative VAS & $1.214(1.035-1.425)$ & 0.017 & NA & \\
\hline Preoperative $\mathrm{Hb}(\mathrm{g} / \mathrm{L}$ ) & $0.974(0.956-0.991)$ & 0.003 & NA & \\
\hline Preoperative ALB (g/L) & $1.018(0.963-1.075)$ & 0.53 & NA & \\
\hline ASA (\%) & $1.432(0.877-2.338)$ & 0.151 & NA & \\
\hline Operation time (min) & $1.014(0.997-1.030)$ & 0.101 & NA & \\
\hline Anesthesia time (min) & $1.018(1.001-1.034)$ & 0.032 & NA & \\
\hline Intraoperative infusion volume (ml) & $2.032(1.709-2.355)$ & $<0.001$ & $1.798(1.645-1.951)$ & 0.028 \\
\hline Intraoperative blood loss (ml) & $1.305(1.185-1.425)$ & $<0.001$ & $1.135(1.068-1.202)$ & 0.008 \\
\hline Out of bed activities time (h) & $1.151(1.074-1.233)$ & 0.028 & NA & \\
\hline Postoperative infusion volume (ml) & $1.007(1.001-1.012)$ & 0.013 & NA & \\
\hline Total infusion volume on the day of surgery ( $\mathrm{ml}$ ) & $1.004(1.002-1.007)$ & 0.001 & NA & \\
\hline VAS score on the day of surgery & $1.540(1.062-2.232)$ & 0.023 & NA & \\
\hline Day 1 after surgery VAS score & $1.512(1.138-2.009)$ & 0.004 & NA & \\
\hline $\mathrm{POD} \mathrm{Hb}(\mathrm{g} / \mathrm{L})$ & $0.951(0.928-0.975)$ & $<0.001$ & $0.955(0.924-0.987)$ & 0.006 \\
\hline POD ALB (g/L) & $0.548(0.481-0.615)$ & $<0.001$ & $0.689(0.656-0.722)$ & 0.026 \\
\hline POD CRP (mg/L) & $0.971(0.917-1.029)$ & 0.316 & NA & \\
\hline POD IL 6 (pg/ml) & $1.485(1.268-1.739)$ & $<0.001$ & $1.312(1.063-1.620)$ & 0.011 \\
\hline POD total blood loss 1 day after surgery (ml) & $1.002(0.997-1.007)$ & 0.424 & NA & \\
\hline POD Hb change rate (\%) & $1.077(1.038-1.118)$ & $<0.001$ & NA & \\
\hline
\end{tabular}

LOS > 48 h group; THA: total hip arthroplasty; ERAS: enhanced recovery after surgery; BMI, body mass index; VAS: visual analogue score; Hb: hemoglobin; ALB: albumin; ASA: American Society of Anesthesiologists; CRP: C-reactive protein; IL-6: interleukin-6; POD: one day after surgery. OR: odds ratio; CI, confidence interval; NA, not available

POD Hb change $=($ Preoperative $\mathrm{Hb}-\mathrm{POD} \mathrm{Hb}) /$ Preoperative $\mathrm{Hb} * 100 \%$

nomogram in a range of risk thresholds (0.03 to 1.00) was higher than that of all-screening or no-screening strategies.

\section{Discussion}

LOS is an essential indicator for assessing the effectiveness of ERAS implementation in developed countries, 


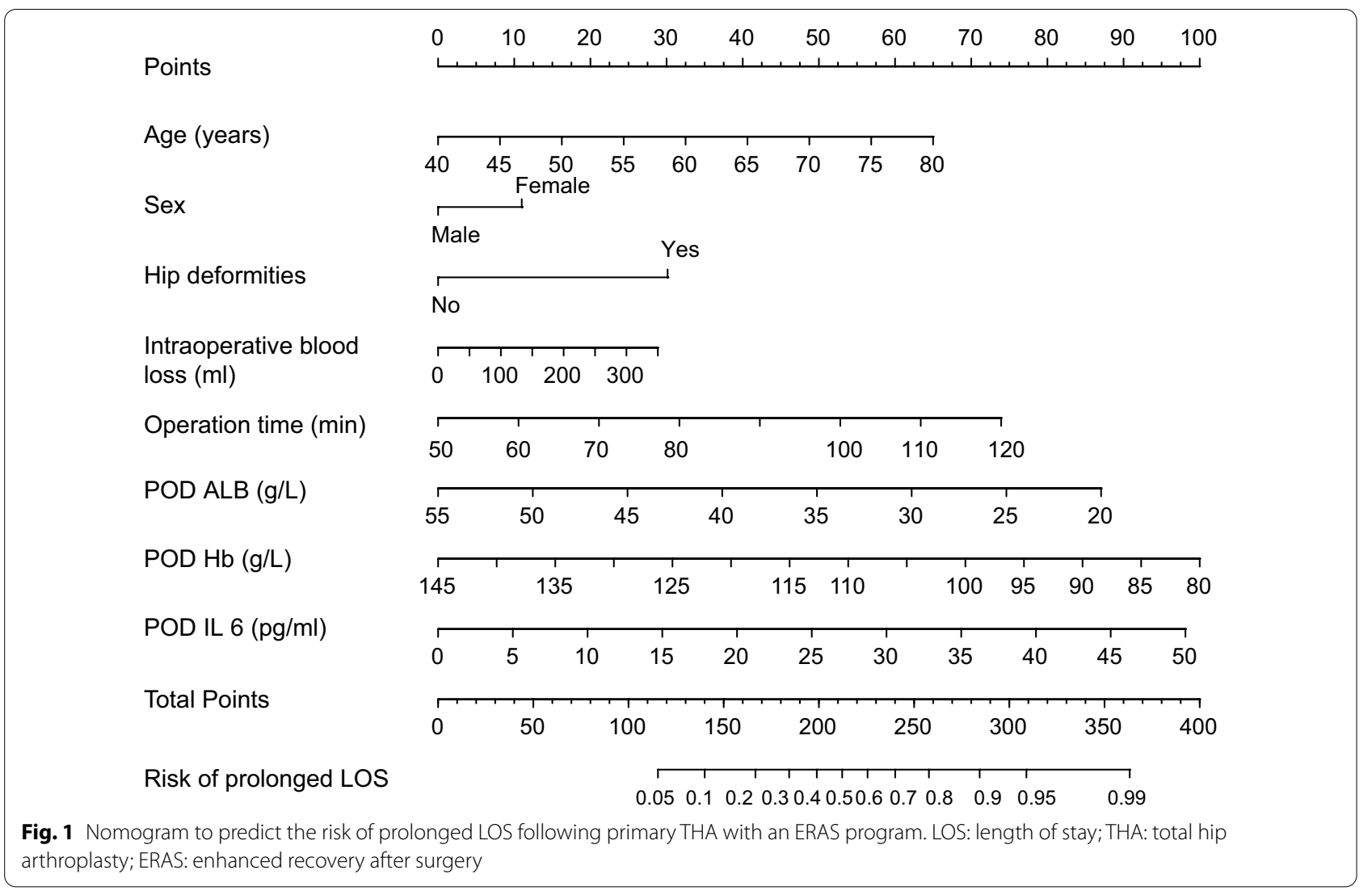

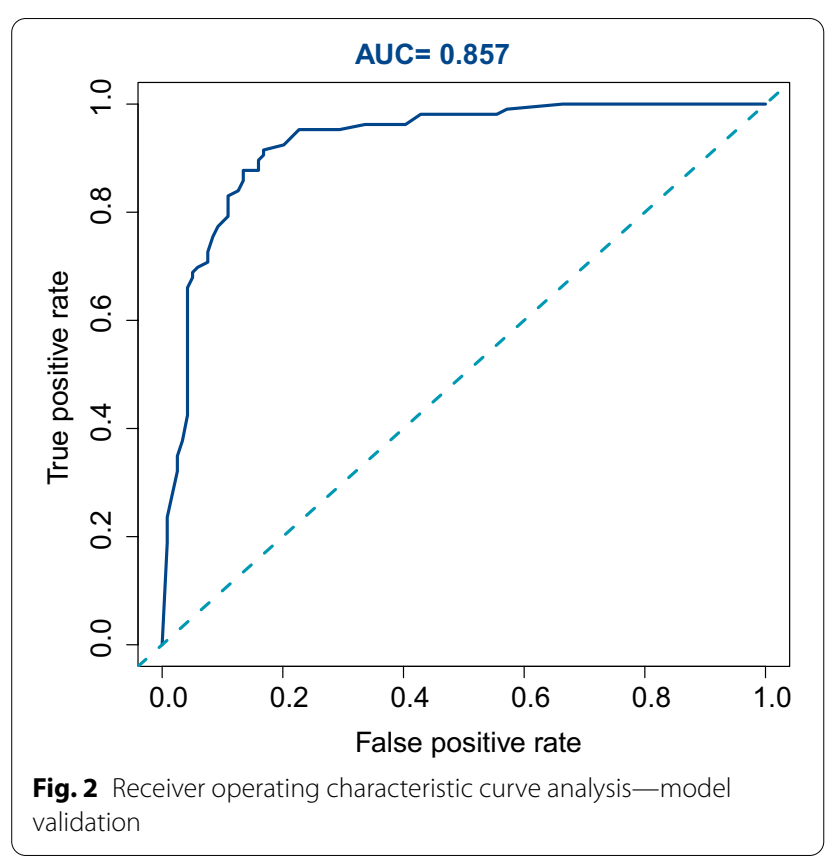

such as in Europe and America [4, 11]. The ERAS program was initially introduced in Denmark, although its use has slowly become more widespread in Europe and

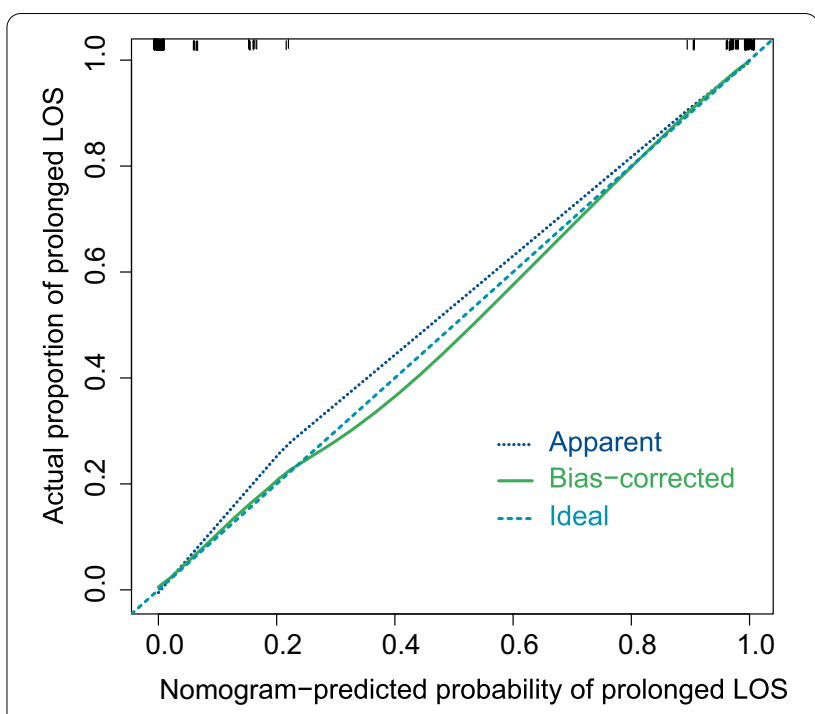

Fig. 3 Calibration plot for the nomogram. The apparent and bias corrected values are close to each other, which means that the nomogram has good predictive performance

America. The total LOS of THA and TKA patients has been reduced from 4-12 $d$ in the past to 2-4 $d$ now, which significantly accelerates the postoperative recovery 


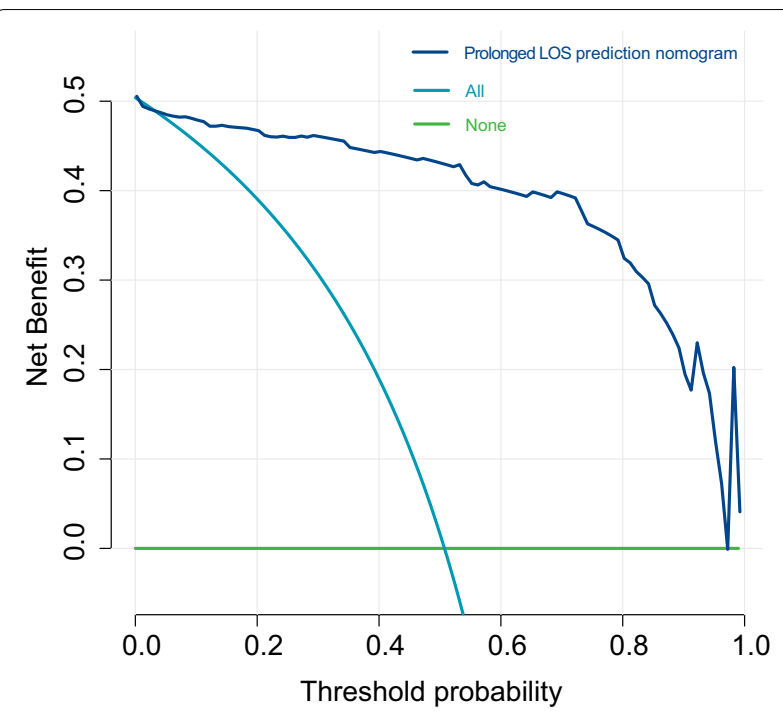

Fig. 4 The decision curve analysis (DCA) for the constructed radiomics nomogram model. The $X$ and $Y$ axes represent the threshold probability and net benefit, respectively. Dark blue indicates the hypothesis that all patients had prolonged LOS. The blue line represents the hypothesis that no patients had prolonged LOS

of patients, saves medical resources, reduces the risk of surgery and improves patient safety and satisfaction [12, 13]. Due to a lack of a comprehensive hierarchical diagnosis and treatment system and relatively adequate medical resources, preoperative examination and preparation are refined by family doctors, community hospitals, or outpatient clinics, and most patients are admitted on the day of surgery and transferred soon after surgery to a rehabilitation facility with the necessary accelerated rehabilitation measures $[14,15]$. Thus, the LOS may not reflect the total LOS but the postoperative LOS.

Compared with developed countries, patients in China tend to visit top-level hospitals without having adequate preoperative workup due to the uneven distribution of medical resources [16]. The process of standardized diagnosis and treatment has been challenging due to the shortage of medical resources, which has resulted in longer preoperative preparation times. Of potentially greater concern, perhaps, is that most patients have difficulty obtaining adequate referrals for rehabilitation after discharge and are instead discharged home directly [17]. Despite the implementation of ERAS protocols in many medical centers, their effect on reducing LOS remains limited. However, little is known about the possible risk factors for prolonged LOS following primary THA with an ERAS program, and a relatively reliable tool for predicting prognosis is lacking. Thus, we screened and identified risk factors for prolonged LOS and developed a nomogram based on a retrospective study. Bootstrapping was the preferred approach for internal validation. In this work, we identified risk factors associated with prolonged LOS as follows: age, sex, hip deformities, intraoperative blood loss, operation time, POD Hb, POD ALB, and POD IL-6.

A total of 392 patients who achieved consensus were included in the present study, with a mean postoperative LOS of $3.4 \pm 1.5 \mathrm{~d}$. Moreover, there was no mortality, and no serious postoperative complications occurred. The patients recovered well after the operation. During the follow-up 3 months after the operation, the excellent and good Harris score rate was 100\% (392/392). In this study, $51.79 \%$ (203/392) of patients were discharged within $48 \mathrm{~h}$ after surgery. The point of $48 \mathrm{~h}$ postoperative was set because most of the literature reported LOS $\leq 2$ $\mathrm{d}$ as an important assessment index of THA in the ERAS program, and the patients in their study were admitted on the day of surgery.

Previously, several studies reported that age is a significant predictor for THA/TKA LOS. In a study reported by Sibia et al. [18], the proportion of patients undergoing THA with a strictly implemented ERAS protocol with a prolonged LOS of more than $1 \mathrm{~d}$ was up to 1.8 times greater in patients aged 70 years than in those aged 60 years. We cautiously speculate that this may be related to the fact that elderly patients have more comorbidities, are in a relatively poorer general condition, and require more care. ElBitar et al. [19] reviewed the data of patients undergoing THA under ERAS implementation and found that patients over 65 years of age and especially over 80 years of age had a prolonged LOS compared to patients under 65 years of age. We believe the possible reason for this is the slower functional recovery after major surgery in the elderly, which prolongs LOS. Despite this lack of evidence, the study identified sex as a risk factor for prolonged LOS in this study. Previous studies have reported that there was an association between female patients and prolonged LOS in THA/TKA [20, 21]. Katz et al. [22] a questionnaire survey found that female patients had a relatively more advanced disease state and poorer lower limb function at the time of surgery, which may have contributed to the longer recovery time required for female patients.

Interestingly, in this report, preoperative combined hip deformities were identified as a risk factor for prolonged LOS, which was similar to the conclusion of the study by Zhang et al. [23]. We cautiously speculate that this may be because this type of patient had a long relative history, and the visits often occurred at an advanced stage of the disease. In addition, a proportion of patients had poor muscle strength in the lower limbs, which caused recovery to be relatively difficult and lead to a longer surgery 
time, thus leading to a slow recovery after surgery and prolonged LOS. The ASA classification and Charlson Comorbidity Index (CCI) are widely accepted parameters for the assessment of comorbidities. Many previous studies have shown that a higher number of comorbidities in patients undergoing THA/TKA corresponds to a higher the risk of prolonged LOS [14, 24, 25]. However, it is worth noting that several issues must be treated with caution such as how to include the types of comorbidities and how to measure the number of comorbidities. Such issues are controversial and will require further study in the future. Therefore, it is important to remain cautious.

In this investigation, intraoperative indicators such as intraoperative blood loss and operation time were identified as risk factors for LOS $>48 \mathrm{~h}$ after THA with an ERAS program. In general, intraoperative transfusion volume and intraoperative blood loss were positively correlated with operation time. More importantly, most of these conditions are more challenging for postoperative recovery because of they are associated with more complex surgery, more bleeding, and increased intraoperative transfusion volume. Previous studies have shown that as the duration of surgery increases, the risk of prolonged LOS also increases [26]. Therefore, according to the designed nomogram, we believe that skilled surgical techniques occupy an important position in reducing LOS and optimizing the surgical operation technique to minimize the operation time is beneficial to the patient's postoperative recovery and reduces LOS. Similarly, we observed that POD Hb was identified as a risk factor for prolonged LOS. This point is easy to understand because POD $\mathrm{Hb}$ often accurately reflects blood loss during the operation. A lower POD Hb indicates a higher perioperative blood loss. Therefore, prompt postoperative review of blood work and intervention at the appropriate time is warranted.

Certain postoperative laboratory parameters, including POD ALB and POD IL 6, were identified as risk factors for prolonged LOS. Patients with low postoperative ALB levels are at higher risk of wound exudation, hematoma, and poor wound healing, resulting in late bedtime activity and poor exercise outcomes. Several studies have confirmed that the late start of functional exercise of the lower extremity after surgery and poor functional exercise of the lower extremity in the early postoperative period are risk factors for prolonged LOS in patients with THA/TKA $[26,27]$. This may be the reason for the prolonged LOS due to low ALB levels on postoperative Day 1. Another unexpected finding of our study was that POD IL 6 was identified as a risk factor for prolonged LOS. IL 6 is a major inflammatory cytokine regulated by $\mathrm{HuR}$ binding to mRNA. Interleukin (IL)-6 is a major inflammatory cytokine regulated by $\mathrm{HuR}$ binding to mRNA. In previous studies IL 6 was considered to be an important proinflammatory factor in cells [27]. Although IL 6 extensively involved in many pathophysiological processes, few papers have reported its effect on LOS after THA. We speculate that surgery and anesthesia may cause elevated IL 6; however, clarifying the molecular biological mechanism will require a large number of future studies to verify this conclusion.

Data on complications indicated that postoperative complications had important effects on prolonged LOS, which suggests that complication prevention, early diagnosis, and appropriate treatment measures have an important impact on clinical outcomes. Although complications were not the most important predictors included in the nomogram model, complications are certainly an important factor worthy of separate study in the future.

Here, we designed a model for predicting the risk of prolonged LOS following primary THA with an ERAS program. The nomogram can demonstrate the key parameters graphically and individually to access the incidence of risk of prolonged LOS. Such work will enable accurate patient evaluation and management when encountered in clinical practice. More importantly, the nomogram may provide opportunities for improving perioperative management and the ERAS program in primary THA.

Various limitations were observed in this investigation. First, the investigation included a retrospective data analysis, which might include unknown confounders, meaning that selection and detection bias cannot be completely avoided. Second, there was a lack of external validation for our proposed model, especially in other regions and countries. Third, the ERAS program followed in this study has only been applied in some parts of China; thus, more collaborative studies in more centers are needed to validate it in the future. Finally, we should focus on the impact of complications on prolonged LOS.

\section{Conclusions}

Overall, this study developed a nomogram tool for predicting prolonged LOS following primary THA with an ERAS program. However, the designed cannot effectively discriminate patients with different prolonged LOS risks. The tool has great potential to aid surgeons in stratifying patient risk and provides a reference for improving ERAS programs. The performance of the nomogram was validated using bootstrap method. The developed nomogram is purely academic thus far; however, we plan to integrate it into the information system of our hospital for prospective validation. 


\section{Abbreviations}

THA: Total hip arthroplasty; ERAS: Enhanced recovery after surgery; BMI: Body mass index; VAS: Visual analogue score; Hb: Hemoglobin; ALB: Albumin; ASA: American Society of Anesthesiologists; CRP: C-reactive protein; IL-6: Interleukin-6; POD: One day after surgery; AUC: Area under the receiver operating characteristic curve; POD: One day after surgery; PONA: Postoperative nausea and vomiting; DVT: Deep vein thrombosis; OR: Odds ratio; Cl: Confidence interval; NA: Not available.

\section{Acknowledgements}

Not applicable.

\section{Authors' contributions}

HSW collected the data, analyzed the data, and drafted the manuscript. MYY supervised the project and reviewed the manuscript. BY, WLL, TTF, and QL conceived of the study, participated in its design and coordination, and helped to draft the manuscript. MYY was responsible for the whole project, designed the study, and supervised the study. All authors read and approved the final manuscript.

\section{Funding}

No funds were received in support of this work.

\section{Availability of data and materials}

The dataset supporting the conclusions of this article is available on request from the corresponding author.

\section{Declarations}

\section{Ethics approval and consent to participate}

The study was approved by an institutional ethics committee at the Taizhou Central Hospital (Project ID: 202009795N). Considering that this work was a retrospective study: the ethics committee waived the requirement for informed consent from patients.

\section{Consent for publication}

Not applicable.

\section{Competing interests}

The authors declare that they have no competing interests.

\section{Author details}

${ }^{1}$ Department of Orthopedics, Taizhou Central Hospital (Affiliated Hospital To Taizhou College), Donghai Street, Taizhou 317700, Zhejiang Province, People's Republic of China. ${ }^{2}$ Department of Endocrinology, Baoji City Hospital of Traditional Chinese Medicine, Baoji, Shaanxi Province, People's Republic of China. ${ }^{3}$ Department of Orthopedics, Xianyang Central Hospital, Xianyang, People's Republic of China. ${ }^{4}$ Clinical Medical Research Center, Xianyang Central Hospital, Xianyang, People's Republic of China. ${ }^{5}$ Department of Orthopedics, Baoji City Hospital of Traditional Chinese Medicine, Baoji, Shaanxi Province, People's Republic of China.

Received: 30 September 2021 Accepted: 5 December 2021 Published online: 14 December 2021

\section{References}

1. Ibrahim M, Twaij H, Giebaly D, Nizam I, Haddad F. Enhanced recovery in total hip replacement: a clinical review. Bone Joint J. 2013;95(12):1587-94.

2. Kent M, Calvert N, Blades K, Swann A, Yates P. Enhanced recovery principles applied to revision hip and knee arthroplasty reduces length of stay and blood transfusion. J Orthop. 2017;14(4):555-60.

3. Tucker A, McCusker D, Gupta N, Bunn J, Murnaghan M. Orthopaedic enhanced recovery programme for elective hip and knee arthroplastycould a regional programme be beneficial? Ulst Med J. 2016;85(2):86.

4. Wainwright TW, Gill M, McDonald DA, Middleton RG, Reed M, Sahota $\mathrm{O}$, Yates $\mathrm{P}$, Ljungqvist $\mathrm{O}$. Consensus statement for perioperative care in total hip replacement and total knee replacement surgery: Enhanced
Recovery After Surgery (ERAS((R))) Society recommendations. Acta Orthop. 2020;91(1):3-19.

5. Joshi GP, Kehlet H. Enhanced recovery pathways: looking into the future. In.: LWW; 2019.

6. Bin S, Xisheng W, Blade L, Tiebing Q, Xianlong Z, Li C, Shigui Y, Liao W-M, Qirong Q, Zhenan Z, et al. Accelerated rehabilitation of hip and knee arthroplasty in China-expert consensus on perioperative pain and sleep management. Chin J Bone Joint Surg. 2016;9(2):91-7.

7. Shaoyun Z, Guorui C, Fuxing P. Perioperative fluid therapy protocol for accelerated rehabilitation of hip and knee arthroplasties. Chin J Orthopaedic Surg. 2018;26(3):234-7.

8. Zongke Z, Xisheng W, Bing X, Tiebing Q, Xianlong Z, Peifu T, Haisan W, Zhenan Z, Weiming L, Qirong Q, et al. Accelerated rehabilitation of hip and knee arthroplasty in China-expert consensus on the diagnosis and treatment of perioperative anemia. Chin J Bone Joint Surg. 2016;9(1):10-5.

9. Zongke Z, Xisheng W, Tiebing Q, Xianlong Z, Shigui Y, Li C, Zhenan Z, Liao W-M, Qirong Q, Liu Y, et al. Accelerated rehabilitation of hip and knee arthroplasty in China-expert consensus on perioperative management strategies. Chin J Bone Joint Surg. 2016;9(1):1-9.

10. Robinson KP, Wagstaff KJ, Sanghera S, Kerry RM. Postoperative pain following primary lower limb arthroplasty and enhanced recovery pathway. Ann R Coll Surg Engl. 2014;96(4):302-6.

11. Wainwright TW, Immins T, Antonis JHA, Hartley R, Middleton RG. Enhanced recovery after surgery: concepts and application to total shoulder replacement. Orthop Nurs. 2019;38(6):375-80.

12. Ripolles-Melchor J, Abad-Motos A, Diez-Remesal Y, AseguinolazaPagola M, Padin-Barreiro L, Sanchez-Martin R, Logrono-Egea M, Catala-Bauset JC, Garcia-Orallo S, Bisbe E, et al. Association between use of enhanced recovery after surgery protocol and postoperative complications in total hip and knee arthroplasty in the postoperative outcomes within enhanced recovery after surgery protocol in elective total hip and knee arthroplasty study (POWER2). JAMA Surg. 2020;155(4):e196024

13. Vendittoli P-A, Pellei K, Desmeules F, Massé V, Loubert C, Lavigne M, Fafard J, Fortier L-P. Enhanced recovery short-stay hip and knee joint replacement program improves patients outcomes while reducing hospital costs. Orthop Traumatol Surg Res. 2019;105(7):1237-43.

14. Deng QF, Gu HY, Peng WY, Zhang Q, Huang ZD, Zhang C, Yu YX. Impact of enhanced recovery after surgery on postoperative recovery after joint arthroplasty: results from a systematic review and meta-analysis. Postgrad Med J. 2018;94(1118):678-93.

15. Huang $Z Y$, Huang Q, Wang LY, Lei YT, Xu H, Shen B, Pei FX. Normal trajectory of Interleukin- 6 and C-reactive protein in the perioperative period of total knee arthroplasty under an enhanced recovery after surgery scenario. BMC Musculoskelet Disord. 2020;21(1):264.

16. Liu H, Liu J, Chen M, Tan X, Zheng T, Kang Z, Gao L, Jiao M, Ning N, Liang $L$, et al. Sleep problems of healthcare workers in tertiary hospital and influencing factors identified through a multilevel analysis: a crosssectional study in China. BMJ Open. 2019;9(12):e032239-e032239.

17. Halawi MJ, Vovos TJ, Green CL, Wellman SS, Attarian DE, Bolognesi MP. Preoperative predictors of extended hospital length of stay following total knee arthroplasty. J Arthroplasty. 2015;30(3):361-4.

18. Sibia US, MacDonald JH, King PJ. Predictors of hospital length of stay in an enhanced recovery after surgery program for primary total hip arthroplasty. J Arthroplasty. 2016;31(10):2119-23.

19. El Bitar YF, Illingworth KD, Scaife SL, Horberg JV, Saleh KJ. Hospital length of stay following primary total knee arthroplasty: data from the nationwide inpatient sample database. J Arthroplasty. 2015;30(10):1710-5.

20. Malek IA, Royce G, Bhatti SU, Whittaker JP, Phillips SP, Wilson IRB, et al. A comparison between the direct anterior and posterior approaches for total hip arthroplasty: the role of an 'Enhanced Recovery' pathway. Bone Joint J. 2016;98(6):754-60.

21. Ibrahim MS, Alazzawi S, Nizam I, Haddad FS. An evidence-based review of enhanced recovery interventions in knee replacement surgery. Ann R Coll Surg Engl. 2013;95(6):386-9.

22. Katz JN, Wright EA, Guadagnoli E, Liang MH, Karlson EW, Cleary PD. Differences between men and women undergoing major orthopedic surgery for degenerative arthritis. Arthritis Rheum. 1994;37(5):687-94. 
23. Zhang S, Huang Q, Xie J, Xu B, Cao G, Pei F. Factors influencing postoperative length of stay in an enhanced recovery after surgery program for primary total knee arthroplasty. J Orthop Surg Res. 2018;13(1):1-7.

24. Tucker A, McCusker D, Gupta N, Bunn J, Murnaghan M. Orthopaedic enhanced recovery programme for elective hip and knee arthroplastycould a regional programme be beneficial? Ulster Med J. 2016;85(2):86.

25. Zhu S, Qian W, Jiang C, Ye C, Chen X. Enhanced recovery after surgery for hip and knee arthroplasty: a systematic review and meta-analysis. Postgrad Med J. 2017;93(1106):736-42.

26. Girbino KL, Klika AK, Barsoum WK, Bloomfield MR, Briskin IN, Brooks PJ, Higuera CA, Kamath AF, Krebs VE, McLaughlin JP. Understanding the main predictors of length of stay after total hip arthroplasty: patient-related or procedure-related risk factors? J. Arthroplasty 2021;36(5):1663-1670. e1664.

27. De Benedetti F, Massa M, Pignatti P, Albani S, Novick D, Martini A. Serum soluble interleukin 6 (IL-6) receptor and IL-6/soluble IL-6 receptor complex in systemic juvenile rheumatoid arthritis. J Clin Investig. 1994;93(5):2114-9.

\section{Publisher's Note}

Springer Nature remains neutral with regard to jurisdictional claims in published maps and institutional affiliations.

- fast, convenient online submission

- thorough peer review by experienced researchers in your field

- rapid publication on acceptance

- support for research data, including large and complex data types

- gold Open Access which fosters wider collaboration and increased citations

- maximum visibility for your research: over 100M website views per year

At BMC, research is always in progress.

Learn more biomedcentral.com/submissions 